\title{
Ocular and neurodevelopmental features of Duchenne muscular dystrophy: a signature of dystrophin function in the central nervous system
}

\author{
Valeria Ricotti ${ }^{1}$, Herbert Jägle ${ }^{2}$, Maria Theodorou ${ }^{3}$, Anthony T Moore ${ }^{3}$, Francesco Muntoni ${ }^{1}$ and \\ Dorothy A Thompson*,3
}

\begin{abstract}
Multiple isoforms of dystrophin (Dp427, Dp260, Dp140, Dp71) are expressed differentially in the central nervous system (CNS) including the retinal layers. Disruption of these protein products is responsible for cognitive dysfunction, electroretinogram (ERG) abnormalities and behavioural disorders in Duchenne muscular dystrophy (DMD). We studied the ocular characteristics and neuropsychiatric profile of 16 DMD boys. The ISCEV standard, full-field flash ERGs were assessed. Intellectual ability and behavioural disturbances were measured. All genotypes were associated with mildly abnormal photopic ERG a:b-wave amplitude ratios. In addition, we identified the following genotype/phenotype correlations: boys with mutations upstream of exon 30 (ie, isolated Dp427 altered expression) showed normal scotopic a:b ratios, abnormal photopic oscillatory potential OP2 and normal scotopic OP2. Conversely, all boys with DMD mutations downstream of exon 30 showed profoundly 'negative' scotopic ERGs (a:b ratios $>1$ ). In these patients, the involvement of Dp260 isoform resulted in the absence of slow rod pathway signalling in $15 \mathrm{~Hz}$ scotopic flicker ERGs. These boys had abnormal scotopic OP2 and normal photopic OP2. Finally, children with mutations also affecting Dp71 were associated with more pronounced electronegative ERGs. When correlating ERGs to neurodevelopmental outcome, we found a positive correlation between negative scotopic ERGs and neurodevelopmental disturbances, and the most severe findings were in boys with Dp71 disruption. These findings suggest a strong association between DMD mutations affecting different DMD isoforms with characteristically abnormal scotopic ERGs and severe neurodevelopmental problems. The role of the ERG as a potential biomarker for dystrophin function in the CNS and response to novel genetic therapies warrants further exploration.

European Journal of Human Genetics (2016) 24, 562-568; doi:10.1038/ejhg.2015.135; published online 17 June 2015
\end{abstract}

\section{INTRODUCTION}

Duchenne muscular dystrophy (DMD) is a life-limiting X-linked recessive disorder affecting 1 in 5000 live male births. ${ }^{1,2}$ It causes progressive muscle weakness, leading to death in the third decade..$^{3-5}$ DMD results from mutations in the dystrophin (DMD) gene on chromosome Xp21.2. The DMD gene is a very large gene with 79 exons and tightly regulated internal promoters, which make a range of protein isoforms, identical at the $\mathrm{C}$ terminus, but with unique $\mathrm{N}$ termini. These include three full-length dystrophin isoforms (Dp427) and the shorter products Dp260, Dp140, Dp116 and Dp71. In the muscle, dystrophin is a large sarcolemmal protein, part of the dystrophin-associated protein complex, connecting the cytoskeleton to the extracellular matrix. ${ }^{6}$ A major function of dystrophin in the muscle is to protect muscle fibres against the mechanical forces of contraction, and thus its absence renders muscle fibres susceptible to stretch-induced damage and necrosis. ${ }^{7}$ Dystrophin has also been assigned a signalling role; although some of these activities are relevant for skeletal muscle function (eg, modulation of adrenergic tone by nNOS), ${ }^{8}$ others are essential for dystrophin function in organs such as the brain and retina. ${ }^{9,10}$ Furthermore, different dystrophin isoforms are localised in distinct regions of the central nervous system (CNS) and the retina. Absence of dystrophin from these sites is responsible for the extraneuromuscular manifestations of DMD including neurodevelopmental disorders ${ }^{11}$ and disturbances in retinal electrophysiology. ${ }^{12}$ Dp140 and Dp71 are largely expressed in the brain, and the lack of both these isoforms is well recognised to be implicated with higher incidence of cognitive impairment and neurodevelopmental disorders in patients with mutations downstream of exon $45 \cdot{ }^{13-15}$ All dystrophin isoforms, with the exception of Dp116, are expressed highly in the retina. Dp427, Dp260 and Dp140 are located in photoreceptor terminals, whereas Dp71 is expressed in Müller glia cells. ${ }^{16-20}$ Recently, Dp427, Dp260 and Dp140 expressions have been identified in inner retinal layer neurons as well; Dp427 was expressed proportionately more at cone than rod synapses, in bipolar cells and some amacrine cells. ${ }^{19}$ Mutations downstream of exon 30, which affect Dp260 expression, are associated with an abnormal scotopic electroretinogram (ERG) waveform ${ }^{21-24}$ and some impairment of red-green colour vision. ${ }^{25}$

In light of the recent retinal expression studies, we sought to explore the clinical ERG as a potential biomarker of CNS dystrophin expression associated with different DMD genotypes and recorded ERGs to a wide range of flash strengths in a cohort of individuals with a range of mutations across the $D M D$ gene. A subsidiary, but important, aim was to assess in this population whether a validated

${ }^{1}$ Dubowitz Neuromuscular Centre, UCL Institute of Child Health and Great Ormond Street Hospital for Children, London, UK; ${ }^{2}$ University Eye Clinic, Regensburg, Germany; ${ }^{3}$ Clinical and Academic Department of Ophthalmology, Great Ormond Street Hospital for Children, London, UK

*Correspondence: Dr DA Thompson, Clinical and Academic Department of Ophthalmology, Great Ormond Street Hospital for Children, London WC1N 3JH, UK. Tel: +44 20 7829 0514; Fax: +44 207829 7946; E-mail: Dorothy.Thompson@gosh.nhs.uk

Received 26 September 2014; revised 1 May 2015; accepted 12 May 2015; published online 17 June 2015 
paediatric ERG protocol would provide results comparable to those from a more demanding international standard method. ${ }^{26}$ Finally, we explored a potential relation between ERG and the neuropsychiatric profile using validated psychometric inventories.

\section{METHODS}

\section{Participants}

Sixteen DMD boys attending the neuromuscular outpatient Department at Great Ormond Street Hospital (GOSH) were recruited according to their genotype, ensuring balanced distribution of mutations downstream and upstream of exon 30 (Table 1), and also tolerability of the assessment. The diagnosis was confirmed by finding an out-of-frame-frame mutation in the $D M D$ gene, using multiplex ligation-dependent probe amplification (MLPA). Where DMD deletions or duplications were not identified by MLPA, all 79 exons and the adjacent introns were analysed through PCR amplification and direct sequencing to screen for point mutations. Mutations were classified according to the Leiden Muscular Dystrophy database. $^{27}$
The nomenclature of the report is based on dystrophin references LRG_199t1. Seven boys had mutations (either out-of-frame deletions, or duplications, or nonsense mutations) involving exons 3-13 (affecting expression of Dp427 isoforms); seven boys had mutations involving exons 44-57 (disrupting Dp427, and, according to their location, also Dp260, Dp140 and Dp116); and two boys had mutations downstream of exon 63 (disrupting all dystrophin isoforms, including the shortest Dp71). All patients were fully cooperative and were able to complete the examination, with the exception of the two boys with $D p 71$ mutation who had a profound intellectual disability, which is common for this genotype, ${ }^{15}$ and could only complete the paediatric protocol. The mean age was 11 years (range 4-15 years). All the boys were on prednisolone given at $0.5-0.75 \mathrm{mg} / \mathrm{kg}$ per day daily or intermittently (10 days on:10 days off). ${ }^{3,4,28}$

\section{Experimental protocol}

All patients underwent eye examinations: visual acuity, Ishihara colour vision assessment, intraocular pressure (IOP) measurement, fundoscopy and electrophysiology examination of the retina using ERGs. ERGs were recorded both to international standards ${ }^{29}$ and with a modified paediatric protocol developed

Table 1 General characteristics of the boys recruited

\begin{tabular}{|c|c|c|c|c|c|}
\hline Individual & $\begin{array}{l}\text { Age at } \\
\text { assessment (years) }\end{array}$ & Mutation & $\begin{array}{l}\text { Dystrophin isoforms predicted } \\
\text { to be affected }\end{array}$ & Neurobehavioural problems & $G A I$ \\
\hline 1 & 7 & Deletion exons 3-7 & Dp427 & $\begin{array}{l}\qquad \mathrm{SCDC}=7 \\
\text { Internalising behavioural problems }\end{array}$ & 98 \\
\hline 2 & 10 & Deletion exons 3-7 & Dp427 & $\begin{array}{c}\operatorname{SCDC}=2 \\
\text { No }\end{array}$ & 102 \\
\hline 3 & 15 & Deletion exons 5-7 & Dp427 & $\begin{array}{l}\qquad \mathrm{SCDC}=7 \\
\text { Attention deficit and anxiety }\end{array}$ & 90 \\
\hline 4 & 11 & Deletion exons 8-13 & Dp427 & $\begin{array}{c}\mathrm{SCDC}=4 \\
\text { No }\end{array}$ & 110 \\
\hline 5 & 10 & Deletion exons 3-11 & Dp427 & $\begin{array}{c}\mathrm{SCDC}=4 \\
\text { No }\end{array}$ & 119 \\
\hline 6 & 10 & Deletion exons 8-13 & Dp427 & $\begin{array}{l}\text { SCDC }=16 \\
\text { Inattention, internalising behavioural } \\
\text { problems and ASD }\end{array}$ & 83 \\
\hline 7 & 8 & Duplication exon 2 & Dp427 & $\begin{array}{c}\mathrm{SCDC}=3 \\
\text { No }\end{array}$ & 90 \\
\hline 8 & 13 & Duplication exons 56-57 & $\begin{array}{l}\text { Dp427, Dp260, Dp140, } \\
\text { Dp116 }\end{array}$ & $\begin{array}{l}\mathrm{SCDC}=10 \\
\text { Internalising behavioural problems and anxiety }\end{array}$ & 119 \\
\hline 9 & 8 & Duplication exons 56-57 & $\begin{array}{l}\text { Dp427, Dp260, Dp140, } \\
\text { Dp116 }\end{array}$ & $\begin{array}{c}\mathrm{SCDC}=9 \\
\text { Attention deficit }\end{array}$ & 96 \\
\hline 10 & 8 & Deletion exons 51-54 & Dp427, Dp260, Dp140 & $\begin{array}{c}\qquad \mathrm{SCDC}=9 \\
\text { Internalising behavioural problems }\end{array}$ & 119 \\
\hline 11 & 12 & Deletion exons $48-50$ & Dp427, Dp260, Dp140 & $\begin{array}{c}\mathrm{SCDC}=0 \\
\text { No }\end{array}$ & 116 \\
\hline 12 & 11 & Deletion exon 44 & Dp427, Dp260, Dp140 & $\begin{array}{l}\mathrm{SCDC}=2 \\
\text { No }\end{array}$ & 99 \\
\hline 13 & 14 & Deletion exons 51 & Dp427, Dp260, Dp140 & $\begin{array}{c}\text { SCDC }=1 \\
\text { No }\end{array}$ & 106 \\
\hline 14 & 10 & Deletion exons 49-54 & Dp427, Dp260, Dp140 & $\begin{array}{c}\text { SCDC }=1 \\
\text { Attention deficit }\end{array}$ & 90 \\
\hline 15 & 11 & Deletion exon 70 & $\begin{array}{l}\text { Dp427, Dp260, Dp140, } \\
\text { Dp116, Dp71 }\end{array}$ & $\begin{array}{c}\mathrm{SCDC}=17 \\
\mathrm{ASD}\end{array}$ & N/A (non-verbal) \\
\hline 16 & 4 & Nonsense mutation exon 70 & $\begin{array}{l}\text { Dp427, Dp260, Dp140, } \\
\text { Dp116, Dp71 }\end{array}$ & $\begin{array}{c}\mathrm{SCDC}=16 \\
\text { ASD }\end{array}$ & $\begin{array}{c}\text { N/A (severe speech } \\
\text { delay) }\end{array}$ \\
\hline
\end{tabular}

Abbreviations: ASD, Autistic Spectrum Disorder; 3Di-sv, Parents' Conners 3, Child Behavioural Check List and the short version of the Developmental Diagnostic and Dimensional Interview; GAI, General Ability Index; SCDC, Social and Communication Disorder Checklist.

A total score SCDC $\geq 8$ is suggestive of emotional sociocommunicative behavioural problems; a total SCDC score of $>15$ is invariably associated with a diagnosis of ASD. IQ is expressed as a GAI score and calculated from a truncated version of the WISC-IV. Behavioural problems are reported as meeting the threshold scores in the 3Di-sv.

a Mutations located towards the $5^{\prime}$ end of the gene (ie mutations from exons 1 to 31 ) only affect the long isoforms Dp427. Mutations in the region of exons $31-44$, in addition to disrupting the expression of Dp427 also disrupt Dp260; mutations in the region of exons 45-62 will in addition disrupt both Dp140 and Dp116. The rare mutations downstream of exon 63 will affect all dystrophin products including the shortest Dp71. 
and validated at GOSH, ${ }^{31,30}$ the details of which are found in the appendix. The range of flash luminance presented in the dark and light was extended to more specifically probe slow and fast signalling pathways in the retina. Images of the retinal macula structure and retinal nerve fibre layer thickness were taken when possible with a high-resolution Fourier optical coherence tomography (OCT) (Heidelberg Spectralis, Heidelberg Engineering, Heidelberg, Germany) device. The intellectual quotient (IQ), where possible, was assessed with a truncated version of the Wechsler Intellectual Scales for Children-Fourth Edition (WISC-IV), ${ }^{32}$ which included Vocabulary, Similarities, Matrix Reasoning, Block Design, Digit Span and Letter-Number Sequencing. The IQ was expressed as a General Ability Index (GAI) score. ${ }^{33}$ The boys' families were asked to complete the Social and Communication Disorders Checklist (SCDC). ${ }^{34}$ The SCDC is a validated 12 -item measure for neurodevelopmental disorders. A total score of $\geq 8$ is indicative of neurodevelopmental disturbances, especially in the social communication domain, whereas scores $>15$ are strongly suggestive of Autistic Spectrum Disorder (ASD). ${ }^{35}$ Families who reported behavioural problems were asked to participate in further assessments including: the Developmental, Diagnostic and Dimensional Interview short version (3Di-sv) to assess ASD, ${ }^{36}$ Conners' Parent Rating Scale (Conners 3$)^{37}$ to assess Attention Deficit Hyperactivity Disorder (ADHD); and the Child and Behavioural Check List $(\mathrm{CBCL})^{37}$ to assess internalising and externalising emotional problems.

\section{Statistics}

The amplitudes and peak times of ERG a- and b-waves from the patients were compared with clinical laboratory age-matched normative data for the ISCEV and GOSH protocols expressed as 5th and 95th centiles. The a:bwave amplitude ratios to scotopic and photopic standard flash stimuli obtained with the GOSH and ISCEV recording techniques were compared using a Pearson's correlation. A one-way ANOVA comparing ERGs and SCDC scores between the genotype groups was performed using the GraphPad Prism version 5.00 for Windows, GraphPad Software. ${ }^{38}$ We set a significant $P$-value at $<0.05$.

\section{RESULTS}

The ERG waveform has a negative a-wave owing to the photoreceptors hyperpolarizing in response to light followed by a positive b-wave that depends on signalling between photoreceptors and bipolar cells depolarising the bipolar cell. Across all genotypes, cone photopic b-wave amplitudes were subnormal, especially to high flash luminances, whereas photopic a-wave amplitudes were $\geq 5$ th centile, indicating normal cone photoreceptor function (Figure 1). In contrast scotopic a-wave amplitudes were subnormal falling below the 5th centile in 6/16 (40\%) of tested patients (Figure 2, bottom graph). Individual scotopic ERG a- and b-wave amplitudes are plotted against normative data (Figure 2).

Boys with mutations downstream of exon 30, affecting both Dp427 and Dp260 (individuals 8-16; Table 1), showed subnormal scotopic b-wave amplitudes to all flash strengths. As the amplitude of the preceding a-wave was larger compared with the b-wave, the a:b amplitude ratios $>1$ and the resulting waveform is called electronegative (Figure 1).

Individuals with mutations affecting only Dp427 (individuals 1-7; Table 1) showed milder scotopic b-wave amplitude reduction, with a:b ratios of 1 (normal a:b 1:2) (Figure 1). The only exception was individual 6, who had a deletion of exons 8-13, yet showed a similar scotopic ERG profile to boys with mutations affecting Dp260. Furthermore, scotopic $15 \mathrm{~Hz}$ flicker ERGs showed that patients with mutations affecting Dp260 (individuals 8-16) lacked signals from the slow rod pathway (Supplementary Figure 1).

Boys with mutations affecting all protein products, including Dp71 (individuals 15-16; Table 1), showed the most profoundly electronegative scotopic ERGs (Figure 3). When comparing photopic and
Rod ERGs

DA 3

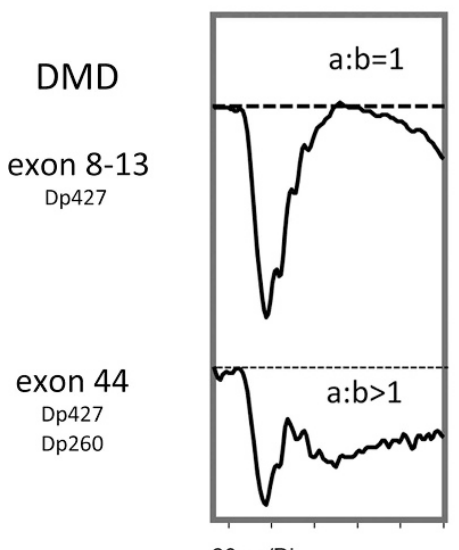

20ms/Div

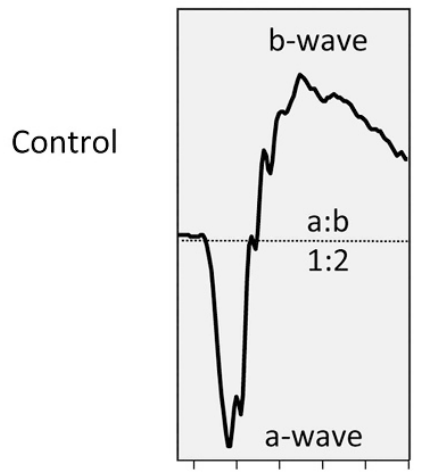

20ms/Div
Cone ERGs

LA 10

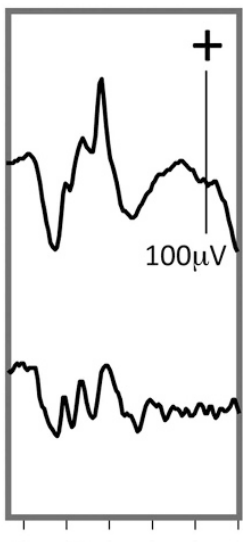

$20 \mathrm{~ms} /$ Div

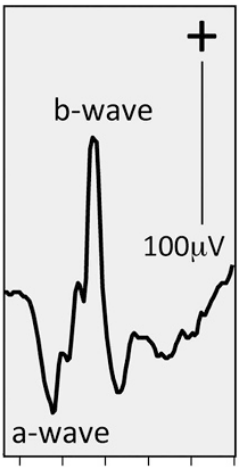

$20 \mathrm{~ms} / \mathrm{Div}$
Figure 1 ISCEV (International Society for Clinical Electrophysiology of Vision) ERG trace examples. Examples of rod and cone ISCEV full-field ERGs from patients with mutations at $5^{\prime}$ and $3^{\prime}$ end of the gene are compared with normal waveforms, in which the a-wave is smaller than the b-wave and the a: $\mathrm{b}$ ratios is $1: 2$. The upper traces from a boy with a mutation affecting Dp427 alone (ie, exons 8-13) show a DA 3 rod ERG in which the b-wave is reduced to the same size as the a-wave giving an a:b ratio of 1 . Below, an individual with a mutation affecting Dp427 and Dp260 (ie, exon 44) with a DA 3 rod ERG b-wave that is smaller than the a-wave giving an electronegative ERG. The cone ERG LA 10 waveforms show a similar pattern with normal a-waves, but small b-wave amplitudes alter the overall ERG waveform.

scotopic mean a:b ratios, we found a significant difference between mutations upstream and downstream of exon $30 \quad(P<0.001)$. Furthermore, the a:b ratio data obtained by the ISCEV corneal electrode protocol were comparable with the GOSH skin electrode protocol ( $r=0.87, P=0.01)$ (Supplementary Figure 2).

The scotopic and photopic ERGs were filtered to reveal four oscillatory potentials (OPs). Boys with Dp427 mutations had a small cone OP2, but normal rod OPs. Those with mutations downstream exon 30 had an absent rod OP2, but surprisingly cone OPs in some were normal (Figure 4).

OCT axial sections performed randomly in six individuals showed normal retinal lamination and macular profiles. The retinal nerve fibre layer thicknesses were within normal limits across genotypes (Supplementary Figure 3).

Clinically, all boys had normal fundi and normal visual acuity. One boy with a nonsense mutation in exon 70 had a red-green colour vision defect. IOPs were mildly elevated in two boys $(22-24 \mathrm{~mm} \mathrm{Hg}$ ). 

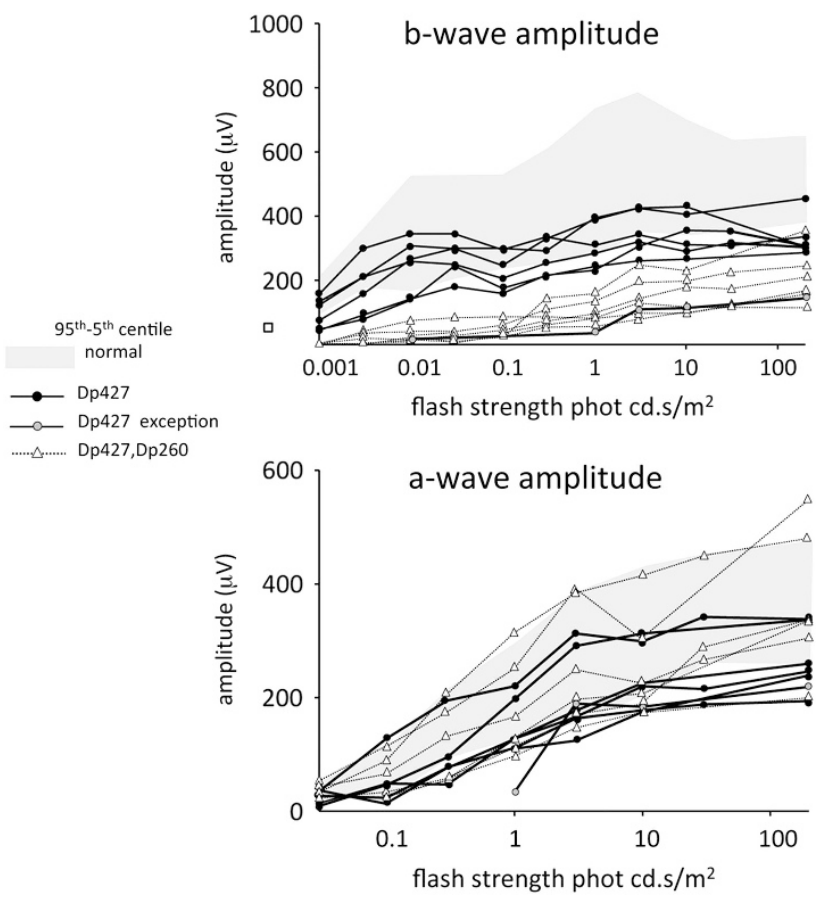

Figure 2 ISCEV (International Society for Clinical Electrophysiology of Vision) scotopic dark-adapted ERG measurements. Top graph: Dark-adapted b-wave amplitude is plotted against flash strength. It shows a dichotomy of Dp427 (filled symbol) and Dp260 (open symbols) response, accentuating the subnormal amplitude when expression of Dp260 is compromised. The b-wave time to peak is also distinctive, falling below the normal range, that is, shorter time to peak, to high increasing flash strength when only the Dp427 expression is affected. Lower graph: a-Wave amplitude is plotted against flash strength. Dp427 (filled symbols) are more often seen below the 5th centile at high flash strengths compared with Dp260 (open symbols), but the time to peak for both fall within the normal range. The exception, shown as a grey filled symbol, is from a patient with deletion of exons 8-13.

\section{Explorative relation between ERG findings, behavioural problems} and intellectual disability

All the subjects recruited in this study were cooperative and with normal range GAI, with the exception of the two boys with Dp71 mutations, who had a profound speech delay. Nine of the 16 boys had neurobehavioural problems: 5/6 boys who scored high in the SCDC had mutations downstream of exon 30 and scored above the threshold for ADHD, ASD and emotional problems (Table 1). Two of seven boys with mutations upstream of exon 30, whose families reported mild behavioural disturbances, had scores above the threshold for inattention and anxiety (Table 1). The two boys with mutations downstream of exon 63 and profound speech delay scored in the ASD range according to both the SCDC and the 3Di-sv. These two boys only managed the GOSH ERG skin protocol.

Interestingly, the same boy (individual 6), with $5^{\prime}$-end mutation, but with a Dp260, $3^{\prime}$-end mutation type of ERG profile also presented with a neurocognitive profile (ie inattention, emotional problems and ASD) comparable to that of children with $3^{\prime}$-end mutations. In this subject, we therefore suspected a second mutation in addition to the deletion of exons 8-13 identified by MLPA, and we carried out a full dystrophin gene sequencing, which however did not reveal other mutations.

Exploring a possible relationship between ERG and neurobehavioural outcomes, we compared the SDCD scores as an overall index

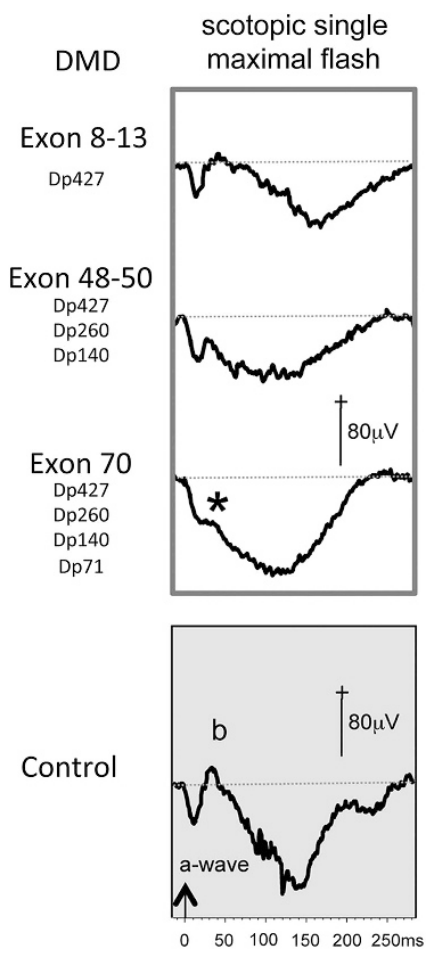

Figure 3 Skin ERG trace examples. Examples of skin ERGs are shown to illustrate responses from individuals with mutations affecting each isoform. The absent b-wave (marked with ${ }^{*}$ ), in association with exon 70 , results in a profoundly electronegative ERG waveform. The trace time base is extended to $250 \mathrm{~ms}$ to show that eventually the polarity of the ERG recovers and the trace rejoins the baseline.

of neurodevelopmental impairment with scotopic a:b ratios. Although our analysis did not meet statistical significance $(P=0.1)$, we observed a trend for boys with electronegative scotopic ERGs more likely to score in the abnormal range for the SDCD (6 out of 10), whereas none of the six boys with electropositive scotopic ERGs had abnormal scores.

\section{DISCUSSION}

We studied the retinal physiology and neurodevelopmental profile of 16 DMD boys, with a range of mutations that affect the different dystrophin isoforms expressed in the CNS and retina (including Dp427, and the shorter isoforms Dp260, Dp140 and Dp71). ${ }^{39}$ We examined the retina functionally with the ERG, and structurally with OCT imaging. We found ERG changes corresponding with the recently reported differential expression of $\mathrm{Dp}$ isoforms between retinal subnetworks in animal models, with Dp427 allied more with cone pathways and Dp260 with rods. ${ }^{19}$

We recognise now that ERG b-wave amplitude mainly depends on depolarisation of on-bipolar cells, ${ }^{40}$ rather than potassium channels on the Müller glial cells, ${ }^{41}$ which are altered in the Dp71-null mouse models of DMD. This explains why ERGs reported in DMD patients differ from some previously predicted DMD mouse knockout models. ${ }^{17,42}$

In our study, DMD patients selectively lacking expression of Dp427 isoforms showed a preponderance of changes in cone b-waves, indicating a disruption of signalling between normally functioning cone photoreceptors and cone on-bipolar cells. In contrast, rod a-wave amplitude was subnormal, indicating altered rod photoreceptor function in 5/7 of patients with mutations affecting Dp427 alone. 


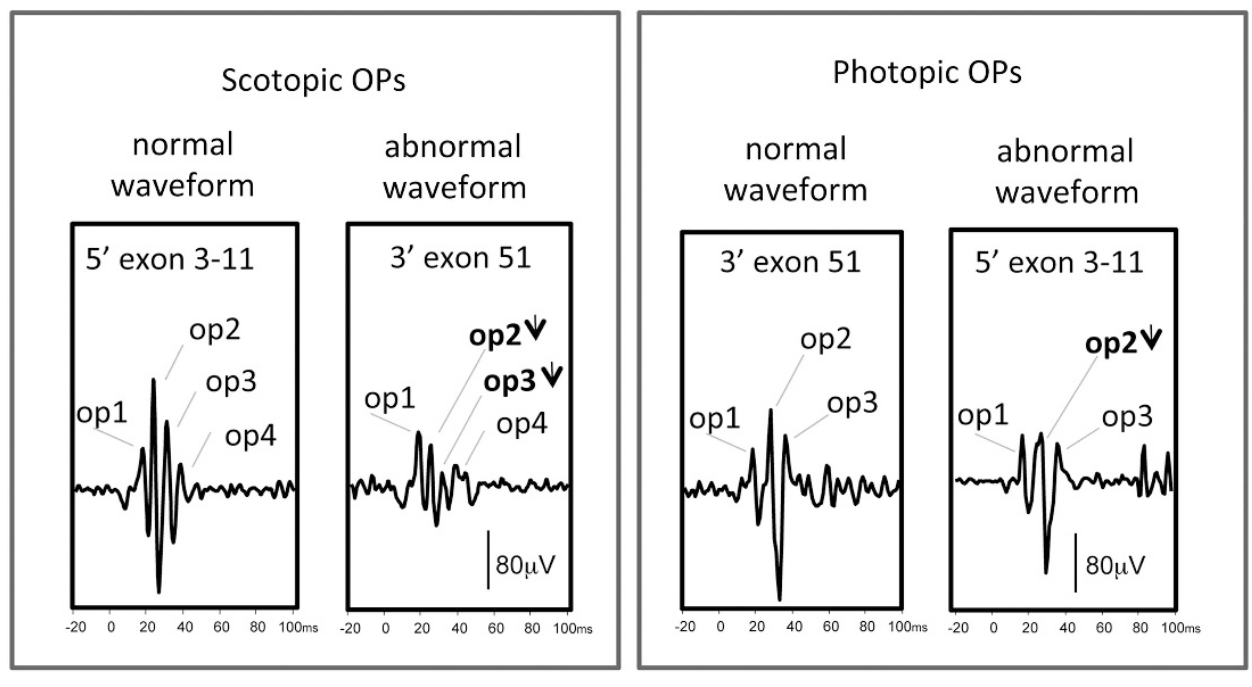

Ribbon synapses bipolars to amacrines

Figure 4 Oscillatory potentials. The OPs are high-frequency wavelets revealed by filtering the ERG trace between 100 and $300 \mathrm{~Hz}$. These are numbered for identification, although loss includes the possibility of OP delay and consequent merger with other OPs. The photopic OP2 in the patient with a mutation at the $5^{\prime}$ end of the gene is subnormal, but the scotopic OPs are normal. Scotopically the greater effect of Dp260 is seen and these scotopic OP2 and OP3 are subnormal, yet unexpectedly this patient has normal photopic OPs.

This has not been reported before and may be due to the brighter flash used in our study. Expression of the Dp260 isoform, between rod photoreceptors and rod on bipolar cells, was necessary for slow rod pathway function. Indeed, altered expression of Dp260 caused subnormal b-wave amplitude and hence electronegative scotopic ERG waveforms. This characteristic signature was exacerbated, if Dp71 was additionally compromised, as shown in Figure 3. In our study, we showed that, indeed, mutations affecting Dp71 have the most profound and uniquely electronegative scotopic ERG (Figure 3), alongside showing most severe cognitive-neuropsychiatric profile disturbances.

Our study highlighted only one exception to these patterns: a boy with a mutation of exons 8-13 (ie affecting Dp427 alone) showed both an electronegative scotopic ERG, severe speech delay and a neuropsychiatric profile in the autistic spectrum domain. We did not identify additional mutations; the combined ERG and neurodevelopmental phenotype of this child remain unexplained.

Retinal signalling depends on the structural alignment of proteins at the synapses. Dystrophin is considered a developmental determinant of structural apposition of photoreceptors with bipolar cells. ${ }^{43} \mathrm{We}$ anticipated that patients with DMD might show altered patterns of retinal lamination; however, high-resolution spectral domain OCT axial images of the in vivo retina did not show any irregularity of the outer plexiform lamina nor abnormal lamina thicknesses in a comparison with normal.

As retinal lamination is normal, the observed ERG changes are more likely due to altered synaptic transmission and membrane polarisation. This implies a role for dystrophin isoforms in retinal signalling. Our novel evidence of isoform-specific loss of rod and cone OPs supports this contention as OPs are generated by spiking amacrine and ganglion cells.

In relation to the neuropsychiatric profile, an important consideration is required: to facilitate electrophysiological assessments, the recruitment was biased towards DMD boys with good understanding and co-operation. With the exception of the two Dp71 boys, who had profound delay, the GAI scores were within normal ranges. Nevertheless, neurobehavioural problems were observed in nine boys and including $\mathrm{ADHD}, \mathrm{ASD}$ and emotional problems. Although three boys with Dp427 mutations presented with some behavioural problems, overall boys with mutations progressively affecting the shorter dystrophin isoforms were more commonly and more severely affected. This is not surprising, as the full-length isoforms are also expressed in the neurocortex, hippocampus and cerebellum. However, the shorter isoforms are most abundantly expressed in the brain, with the pivot region of interest for the brain in exon 45 . Indeed, Dp140 has been amply localised in glial cells, ${ }^{44}$ and disruption of this isoform in mutations affecting exons 45-62, has been implicated with cognitive impairment, higher incidence of ADHD and delayed milestones. ${ }^{13,14,45}$ Mutations affecting Dp71, a brain isoform implicated in transmembrane permeability, ${ }^{46}$ although rare, are recognised in association with severe cognitive impairment. ${ }^{13,47}$ The two boys with Dp71 mutations, indeed, had the most severe profile with ASD and profound speech delay. Our findings, in line with previous reports in the literature, suggest a possible cumulative effect on neurodevelopment caused by the progressive loss of the shorter dystrophin isoforms, with mutations towards the $3^{\prime}$ end of DMD being associated with the most devastating outcomes.

In our cohort, we observed a trend for scotopic electronegative ERGs to be associated with more likelihood of neurodevelopmental disorders. However, the spectrum of neuropsychiatric morbidities in dystrophinopathies is vast, complex and multifactorial; our sample population was relatively small; and our observations were based on validated questionnaires/interviews rather than extensive neuropsychological assessments. Therefore, an association between retinal electrophysiology and neurodevelopmental disturbances needs further exploration in larger cohorts.

Experimental treatments with antisense oligonucleotide-mediated exon skipping or drugs that allow read-through nonsense mutations are currently being tested in human clinical trials, demonstrating successful correction restoration of dystrophin expression in the muscle. ${ }^{48,49}$ A new class of therapeutic agents have recently shown to be able to cross the blood-brain barrier in animal model and also to restore dystrophin in the CNS; ${ }^{50}$ therefore, there is both the need to 
better understand the DMD CNS changes and to identify the treatment response with quantifiable biomarkers.

Our study shows that retinal electrophysiology is a tool sensitive to dystrophin protein absence in the CNS; it provides an individual patient pretreatment retinal response profile as a signature of protein disruption. In the absence of structural defects in the eye of DMD boys, we can speculate that the electrophysiological signature may change towards normal as the protein is also restored in the CNS; however, this model requires further exploration in the retina, whereas it was demonstrated in the brain. ${ }^{51}$ The ophthalmic manifestations of myopathies have recognised importance as potential biomarkers, ${ }^{52}$ and in DMD the retina characteristics may offer a portal to the CNS.

Two boys with severe neurocognitive profile could only manage the GOSH skin protocol. This technique offers a tolerable, child friendly assessment of retinal physiology if the neurobehavioural profile is severely affected, which is often the case in DMD, especially in boys with mutations at the $3^{\prime}$ end of the gene.

In conclusion, our data further refine the characteristics of the retinal profile of DMD. The frequently reported electronegative scotopic ERG associates with mutations downstream of exon 30 and is profound when Dp71 is additionally affected. Selective stimulation of slow and fast rod pathways shows a dependence of slow rod pathways upon Dp260. Also, we have highlighted anomalies of the amacrine OP circuits, and cone pathways associated with Dp427 and Dp71. The most severe b-wave changes reflect the most severe neurocognitive profile and likely abnormal dystrophin isoform CNS expression. Although further investigating the intricate and yet poorly understood role of dystrophin and its isoforms, retinal electrophysiology could offer a noninvasive portal into understanding protein disruption and potential restoration in the CNS.

\section{CONFLICT OF INTEREST}

FM has served on scientific advisory boards for, Prosensa, Servier, Roche, PTC Therapeutics and Pfizer. FM receives research support from PTC Therapeutics, Prosensa, Sarepta Therapeutics, Roche, ISIS and Summit plc. VR, HJ, MT, ATM and DT report no disclosures.

\section{ACKNOWLEDGEMENTS}

The financial support of L'Association Française contre les Myopathies (AFM) is acknowledged (to VR). FM is supported by the Great Ormond Street Hospital Biomedical Research centre. The financial support of the Muscular Dystrophy Campaign to the Dubowitz Neuromuscular Centre is gratefully acknowledged.

\section{AUTHOR CONTRIBUTIONS}

DT, FM, ATM and VR oversaw the conduct of the study and the collection of data. DT, HJ, MT and ATM designed the assessment protocol. VR recruited patients and administered the behavioural questionnaire/interview and cognitive assessment. DT, HJ and VR analysed the data. VR and DT wrote the first draft of the manuscript. VR, HJ, MT, ATM, FM and DT contributed to the revision of the manuscript.

1 Ellis JA, Vroom E, Muntoni F: 195th ENMC International Workshop: Newborn screening for Duchenne muscular dystrophy 14-16th December, 2012, Naarden, The Netherlands. Neuromuscul Disord 2013; 23: 682-689.

2 Appleton RE, Bushby K, Gardner-Medwin D, Welch J, Kelly PJ: Head circumference and intellectual performance of patients with Duchenne muscular dystrophy. Dev Med Child Neurol 1991; 33: 884-890.

3 Bushby K, Finkel R, Birnkrant DJ et al: Diagnosis and management of Duchenne muscular dystrophy, part 2: implementation of multidisciplinary care. Lancet Neurol 2010; 9: 177-189.

4 Bushby K, Finkel R, Birnkrant DJ, et al: Diagnosis and management of Duchenne muscular dystrophy, part 1: diagnosis, and pharmacological and psychosocial management. Lancet Neurol 2010; 9: 77-93.
5 Bushby K, Connor E: Clinical outcome measures for trials in Duchenne muscular dystrophy: report from International Working Group meetings. Clin Invest (Lond) 2011; 1: 1217-1235.

6 Envasti JM: Dystrophin, its interactions with other proteins, and implications for muscular dystrophy. Biochim Biophys Acta 2007; 1772: 108-117.

7 Le Rumeur E, Winder SJ, Hubert JF: Dystrophin: more than just the sum of its parts. Biochim Biophys Acta 2010; 1804: 1713-1722.

8 Brenman JE, Chao DS, Xia H, Aldape K, Bredt DS: Nitric oxide synthase complexed with dystrophin and absent from skeletal muscle sarcolemma in Duchenne muscular dystrophy. Cell 1995; 82: 743-752.

9 Pillers DA, Weleber RG, Green DG, et al: Effects of dystrophin isoforms on signal transduction through neural retina: genotype-phenotype analysis of duchenne muscular dystrophy mouse mutants. Mol Genet Metab 1999; 66: 100-110.

10 Anderson JL, Head SI, Rae C, Morley JW: Brain function in Duchenne muscular dystrophy. Brain 2002; 125: 4-13.

11 Ricotti V, Roberts RG, Muntoni F: Dystrophin and the brain. Dev Med Child Neurol 2011; 53: 12

12 Pillers DA: Dystrophin and the retina. Mol Genet Metab 1999; 68: 304-309.

13 Lorusso ML, Civati F, Molteni M, Turconi AC, Bresolin N, D'Angelo MG: Specific profiles of neurocognitive and reading functions in a sample of 42 Italian boys with Duchenne Muscular Dystrophy. Child Neuropsychol 2013; 19: 350-369.

14 Pane M, Lombardo ME, Alfieri $\mathrm{P}$, et al: Attention deficit hyperactivity disorder and cognitive function in Duchenne muscular dystrophy: phenotype-genotype correlation. J Pediatr 2012; 161: 705-709, e701.

15 Daoud F, Angeard N, Demerre B, et al: Analysis of Dp71 contribution in the severity of mental retardation through comparison of Duchenne and Becker patients differing by mutation consequences on Dp71 expression. Hum Mol Genet 2009; 18: 3779-3794.

16 Ueda H, Baba T, Terada N, Kato Y, Tsukahara S, Ohno S: Dystrophin in rod spherules; submembranous dense regions facing bipolar cell processes. Histochem Cell Biol 1997; 108: 243-248.

17 Dalloz C, Sarig R, Fort $\mathrm{P}$, et al: Targeted inactivation of dystrophin gene product Dp71: phenotypic impact in mouse retina. Hum Mol Genet 2003; 12: 1543-1554.

18 Tadayoni R, Rendon A, Soria-Jasso LE, Cisneros B: Dystrophin Dp71: the smallest but multifunctional product of the Duchenne muscular dystrophy gene. Mol Neurobiol 2012; 45: 43-60.

19 Wersinger E, Bordais A, Schwab Y, et al: Reevaluation of dystrophin localization in the mouse retina. Invest Ophthalmol Vis Sci 2011; 52: 7901-7908.

20 Rodius F, Claudepierre T, Rosas-Vargas $\mathrm{H}$ et al: Dystrophins in developing retina: Dp260 expression correlates with synaptic maturation. NeuroReport 1997; 8: 2383-2387.

21 Pillers DA, Weleber RG, Woodward WR, Green DG, Chapman VM, Ray PN: mdxCv3 mouse is a model for electroretinography of Duchenne/Becker muscular dystrophy. Invest Ophthalmol Vis Sci 1995; 36: 462-466.

22 Pillers DA, Fitzgerald KM, Duncan NM, et al: Duchenne/Becker muscular dystrophy: correlation of phenotype by electroretinography with sites of dystrophin mutations. Hum Genet 1999; 105: 2-9.

23 Kameya S, Araki E, Katsuki M et al: Dp260 disrupted mice revealed prolonged implicit time of the b-wave in ERG and loss of accumulation of beta-dystroglycan in the outer plexiform layer of the retina. Hum Mol Genet 1997; 6: 2195-2203.

24 Barboni MT, Nagy BV, de Araujo Moura AL et al: ON and OFF electroretinography and contrast sensitivity in Duchenne muscular dystrophy. Invest Ophthalmol Vis Sci 2013; 54: 3195-3204.

25 Costa MF, Oliveira AG, Feitosa-Santana C, Zatz M, Ventura DF: Red-green color vision impairment in Duchenne muscular dystrophy. Am J Hum Genet 2007; 80 1064-1075.

26 Marmor MF, Fulton AB, Holder GE, Miyake Y, Brigell M, Bach M: ISCEV Standard for full-field clinical electroretinography (2008 update). Doc Ophthalmol 2009; 118 69-77 http://www.dml.nl (last accessed in September 2014).

27 Center for Human and Clinical Genetics. Leiden Muscular Dystrophy pages. Leiden University Medical Center, 2011. http//www.dml.nl (last accessed in September 2014)

28 Ricotti V, Ridout DA, Scott E et al: Long-term benefits and adverse effects of intermittent versus daily glucocorticoids in boys with Duchenne muscular dystrophy. J Neurol Neurosurg Psychiatry 2012; 84: 698-705.

29 McCulloch DL, Marmor MF, Brigell MG et al: ISCEV Standard for full-field clinical electroretinography (2015 update). Doc Ophthalmol 2015; 130: 1-12.

30 Kriss A: Skin ERGs: their effectiveness in paediatric visual assessment, confounding factors, and comparison with ERGs recorded using various types of corneal electrode. Int J Psychophysiol 1994; 16: 137-146.

31 Thompson DA, Liasis A: Visual Electrophysiology: How Can it Help You and Your Patient. China: Hoyt and Taylor, 2012.

32 Wechsler D: Weschler Intelligence Scale for Children-Fourth Edition (WISC-IV). London, UK: Pearsons Assessment, 2004.

33 Prifitera A, Saklofske D: WISC-III Clinical Use and Interpretation. New York, NY, USA: Academic Press, Elsevier, 1998. pp 1-38.

34 Skuse DH, Mandy WP, Scourfield J: Measuring autistic traits: heritability, reliability and validity of the Social and Communication Disorders Checklist. Br J Psychiatry 2005; 187: 568-572.

35 Skuse DH, Mandy W, Steer $C$ et al: Social communication competence and functional adaptation in a general population of children: preliminary evidence for sex-by-verbal IQ differential risk. J Am Acad Child Adolesc Psychiatry 2009; 48: 128-137.

36 Santosh PJ, Mandy WP, Puura K, Kaartinen M, Warrington R, Skuse DH: The construction and validation of a short form of the developmental, diagnostic and dimensional interview. Eur Child Adolesc Psychiatry 2009. 18: 521-524. 
37 Conners CK, Sitarenios G, Parker JD, Epstein JN: The revised Conners' Parent Rating Scale (CPRS-R): factor structure, reliability, and criterion validity. J Abnorm Child Psychol 1998; 26: 257-268.

38 GraphPad Prism version 5.00 for Windows. GraphPad Software: San Diego, CA, USA, 2005, 5th edn. Available at: http://www.graphpad.com (Last accessed September 2014).

39 Muntoni F, Torelli S, Ferlini A: Dystrophin and mutations: one gene, several proteins, multiple phenotypes. Lancet Neurol 2003; 2: 731-740.

40 Robson JG, Frishman LJ: Dissecting the dark-adapted electroretinogram. Doc Ophthalmol 1998; 95: 187-215.

41 Thompson DA, Feather S, Stanescu HC, et al: Altered electroretinograms in patients with KCNJ10 mutations and EAST syndrome. J Physiol 2011; 589: 1681-1689.

42 Cia D, Simonutti M, Fort PE, Doly M, Rendon A: Slight alteration of the electroretinogram in mice lacking dystrophin dp71. Ophthalmic Res 2014; 51: 196-203.

43 Girlanda P, Quartarone A, Buceti R, et al: Extra-muscle involvement in dystrophinopathies: an electroretinography and evoked potential study. J Neurol Sci 1997; 146: 127-132.

44 Lidov HG, Selig S, Kunkel LM: Dp140: a novel $140 \mathrm{kDa}$ CNS transcript from the dystrophin locus. Hum Mol Genet 1995; 4: 329-335.

45 Pane M, Scalise R, Berardinelli A et al: Early neurodevelopmental assessment in Duchenne muscular dystrophy. Neuromuscul Disord 2013; 23: 451-455.
46 Nicchia GP, Rossi A, Nudel U, Svelto M, Frigeri A: Dystrophin-dependent and -independent AQP4 pools are expressed in the mouse brain. Glia 2008; 56: 869-876.

47 Taylor B, Jick H, Maclaughlin D: Prevalence and incidence rates of autism in the UK: time trend from 2004-2010 in children aged 8 years. BMJ Open 2013; 3: e003219.

48 Goemans NM, Tulinius M, van den Akker JT, et al: Systemic administration of PRO051 in Duchenne's muscular dystrophy. N Engl J Med 2011; 364: 1513-1522.

49 Cirak S, Arechavala-Gomeza V, Guglieri M, et al: Exon skipping and dystrophin restoration in patients with Duchenne muscular dystrophy after systemic phosphorodiamidate morpholino oligomer treatment: an open-label, phase 2, doseescalation study. Lancet 2011; 378: 595-605.

50 Goyenvalle A, Griffith G, Babbs A, et al: Functional correction in mouse models of muscular dystrophy using exon-skipping tricyclo-DNA oligomers. Nat Med 2015; 21: 270-275.

51 Dallerac G, Perronnet C, Chagneau C et al: Rescue of a dystrophin-like protein by exon skipping normalizes synaptic plasticity in the hippocampus of the mdx mouse. Neurobiol Dis 2011; 43: 635-641.

52 Kersten HM, Roxburgh RH, Danesh-Meyer HV: Ophthalmic manifestations of inherited neurodegenerative disorders. Nat Rev Neurol 2014; 10: 349-362.

Supplementary Information accompanies this paper on European Journal of Human Genetics website (http://www.nature.com/ejhg) 